\title{
The Resolution of Musical Chords: A Physical Paradigm
}

\author{
Steve Mathew D A1,2* \\ 1. Indian Institute of Technology Madras, Chennai, IN \\ 2. Vellore Institute of Technology, Vellore, IN \\ Mail: stevemathew.da2020@vitstudent.ac.in
}

In any form of music, the fundamental aspect which gives the most of an essence is the tune of the composition. An integral concept in orchestral music is chords. Chords usually follow the notes of the song, making it harmonious with the overall progression of the performance. Chords are often interchangeable within the scale of the song. The mellifluous effect of chords and the harmony it portrays are self-explanatory and pleasant. There lies a mathematical and physical reason behind the working of these chords and the movement shown by them during the piece. In this study, we look at the fundamental tonal frequencies associated with the notes of the chords and analyze the patterns exhibited and draw meaningful conclusions corroborating the scientific relationship with music and its play, while proposing a new musical phenomenon called 'tonal inertia' that seems to potentially explain the musical conventions using physical bases.

Keywords: musical chords, cognitive musicology, tonal stability, auditory perception, music analysis, mathematical music

\section{Introduction}

Though the state of analyzing music from a musical perspective is adequate, knowing the science behind its working can help a composer explore the dark spheres of music theory and application. This study is conducted based on the hypothesis that, behind the notes of chords that resolve, there lies an underlying reason in their fundamental tonal frequencies that facilitate the harmonic transition. When the same was done, we could sense another basis upon which the chords seemed to go in agreement; the chords tend to remain moving in the same direction as the progress in a song. This is described in this paper as 'tonal inertia'. It is self-explanatory that the music played in a systematic sequence is highly endearing as our brains can predict what is coming up next $(1,2)$. This neurobiological trait is used at our advantage to come up with pleasant compositions.

Till date, many studies have gone into the field of music and its scientific principles of working. The resolution from seventh chords to triads have been conducted involving enharmonic reinterpretation (3). Tonal interference on frequency discrimination of a tonal signal was analyzed (4). Working on the tonal levels has led to decipher the effect of leading-tone on the pitch of a sine tone-intensity (5). These works in turn reflect on the characteristics of musical notes.

\section{Methods}

\section{A. Working of the Leading-tone}

Musicians often find that the most pleasing way to resolve to the tonic or to any sustained note in a piece is through its respective leading-tone. In many written rules of classical music and in contemporary genres, the usage of leading-tone is indispensable. The note tends to be a little unstable and further facilitates a movement to the next immediate semitone. The physical reason for the same can be explained by plotting the frequencies tonic and leading-tone against time using the formula $\mathrm{y}(\mathrm{t})=\sin (2 \pi \mathrm{ft})$. When the same was done, an intricate pattern was found in the relationship of these notes. They both intersected once in every $\sim 0.24$ seconds (as shown in Table. 1 and Figure. 1) accurately at $\sin (t)=0$ (This entire study was conducted with respect to the $D$ 
diatonic major scale; the final conclusions are transferrable to all scales). It is obvious that the more frequent the intersections, the more consonant are the tones since the unison is with a constant overlap with itself and is the most consonant in a given scale. When the other notes of the scale were subjected to the same experiment, they showed smaller intervals. It is also worthwhile to note that some notes showed more than 1 common difference in a mix in their intersections. As far as the leading-tone is concerned, its graph intersects with that of the tonic roughly only 4 times in a second. This rarity makes it thrive to arrive at the tonic as soon as possible. This is the reason for chords that have the leading-tone in them like the major triad of the dominant (perfect cadence), the diminished triad of the leading-tone, the Major 7th Chord of the tonic, 'm7(b5)' of leadingtone resolve to the major of the tonic in a diatonic major scale.

\section{B. Augmenting property of Supertonic in Resolution}

In continuation of the above process, it is also worthwhile to note that the supertonic resolves very well back to the tonic. Using the same steps, as we look at its frequency, we see that the intersections show a pattern of common differences varying as $\sim 0.449, \sim 0.109, \sim 0.109$ (Table. 2 ). When looked closely, we observe that the graph of supertonic also intersects with the tonic only 4 times in a second (though not exactly periodical). Thus, this rarity in intersections coupled with the effect of the leading-tone makes the already discussed chords resolve to tonic harmoniously.

\section{Tonal Inertia}

It is proven that when a piece of music follows a pattern, it enables the listener to predict its result which often seems pleasurable $(1,2)$. Inertia, to quote Sir Isaac Newton, is the tendency of a physical object to stay in its state of motion or rest without the influence of an external force acting upon it (6). Though Newtonian laws of physics are absurd in the field of music, the following proposal has a similar property. The notes when played in a particular sequence, preferably with constant intervals/following a scale in the same direction sound pleasurable. This is the reason why the progression of any conventional scale sounds endearing. Any musical scale when is played in a sequence of increasing/decreasing frequencies, sounds good. It is the reason why conventionally when the scales are played, the notes aren't jumbled. When transposing the same concept into the scope of chords, we can analyze bass lines, or practically any note of a chord and conclude that the chords too sound pleasant when they are progressing either upwards (right up the keyboard) or downwards (left down the keyboard).

When the concept of tonal inertia is applied to the paradigm of resolution by supertonic, a practical complexity occurs; due to the rarity of intersections, the note wants to resolve back to tonic as soon as possible. The distance between the tonic and the supertonic is large when compared with that of the leading-tone and tonic. Thus, it is obvious that the degree of gross resolutive effect of the leading-tone is higher than of the supertonic. Hence supertonic alone cannot help resolving itself to the tonic smoothly whereas it can couple with another resolving note making the progression smooth. As far as the hypothesis of tonal inertia is concerned, the tendency for a flow of notes to stay in a particular direction can facilitate the same in the opposite direction as well i.e. from the high to low notes. Another physical advantage to this aspect is that the notes in the piano follow an exponential increase along the keyboard; the frequencies of subsequent notes increase by a factor of the 12th root of 2 (7). Thus, falling back left from a higher note is easy as the frequency difference would be lower, thus facilitating a shorter leap.

A classic example proving the above theory is the song 'Above All' (8). The marked notes are added ones (Figure. 2) and the remaining notes depict the tune and suitable chords of the song in its simplest form. It can be observed that the notes when made to proceed in the same direction, 
ended up resulting in other meaningful chords that are not diatonic to the scale. In the first addition, the notes came down the scale and stopped at the major $3 \mathrm{rd}$. The chord used there was its major. The reason was to facilitate moving to the succeeding chord, the major of the subdominant. The reason why the major chord of major 3rd works is because, it houses the leading-tone of the scale whose tonic major chord is the destination. Simultaneously, a slight dissonance is given by housing the minor 6th which adds to the necessity to resolve to a stable chord. According to the usual working of leading-tone, the minor 6th ascends to the major 6th as it would happen in the diatonic major scale of the major 6th.

\section{Practical Discussions}

It is worthwhile to note that the scientific inferences from this section agree with the concept of Stable and Active Notes (10).

\section{i. The harmonious disharmony: Minor of Major $3^{\text {rd }}$ and Relative Minor}

As far as genres Rock and Pop are concerned, it is relatively conventional to stick on with the chords and harmony diatonic to the scale, unlike Jazz. The minor chords of the major 3rd and major 6th are soulful and moving chords in the scale. The reason behind the pleasing dissonant tint behind these chords can be explained by the previously discussed properties. As far as the minor of the major 3rd is concerned, the magic is created by the movement that is voluntarily made from the tonic to the leading- tone; it is not a movement that would be sought after in the first place. It requires the most stable note, unison here aka. tonic to move behind to the leadingtone (voicing does have an impact but doesn't significantly affect the dissonance experienced). This goes against the fundamental scientific working rule of the leading-tone. Though the root of the succeeding chord is situated higher than the tonic in the scale, practically the tonic replaced by the leading-tone puts forth a tautological dissonance.

The relative minor works very similar to the one as discussed. Rather than substituting the tonic, here the substitution happens at the second most stable note of the major scale, the dominant.

\section{ii. Fundamental enticement towards Major Chords in a Major Scale}

This topic of study requires a practical example. Here we look at one of the most commonly used chord progressions (9) the $\mathrm{I}-\mathrm{V}-\mathrm{vi}-\mathrm{IV}$ progression. Understanding how the chords move on the keyboard is mutually transferrable and clarity in the same can be applied to any complex chord progression. The 1st and 2 nd notes of the I triad move back in the scale to the previous degree to arrive at the V. This is a voluntary 'push'. The next chord tends to be in the same direction. The 2nd and the 3rd notes (with tonic as first) of the $\mathrm{V}$ triad move 1 degree down the scale and the 1 st note moves 2 notes down the scale. Though the magnitude of tonal inertia in each note varies, the direction is constant. This is the point where the enticement is about to take place. It would be pleasing to propose that in a major scale, the slightest dissonance created by a minor chord can be efficiently resolved only by a major chord and vice-versa. The reason is the physical stability of a major chord when compared to a minor chord. The two notes that are in a triad (apart from the key-center) can be individually graphed against that of the tonic and the common points of intersection in a duration of time can be recorded to find the degree of consonance. When the same was done, it was found that the tonic major here had all its notes in consonance for the first time at 0.054 seconds and the tonic minor triad did so at 0.21 seconds. Thus, the most resolutive effect in a major scale is rendered by the diatonic major chords. In the same manner, in a minor scale where a small tint of dissonance is its significance, the diatonic minor chords resolve in a pleasing way. This fact is still strengthened by a minor piece's tendency to resolve predominantly 
to the minor of the tonic and not its relative major (9). Thus, the minor of the major 6th resolves to the major of the subdominant as it can be achieved by altering just one note (the 3 rd note of the triad) like the way the leading-tone would resolve to the tonic in the diatonic major scale with the major 6th as the tonic.

\section{iii. Flexible nature of the Supertonic}

We had already seen that the super-tonic plays an augmenting role in resolution of chords. Since it does not have a specific target for resolution like that of the leading-tone, supertonic can be coupled with any other resolving note to arrive at the destination chord effectively with an added purpose. It is worthwhile to note that the minor of the supertonic can open up or close down a line of composition making it versatile. This is concluded as it houses the notes supertonic and major 6th which are diatonic yet not the most stable; major 6th undergoes intersection 9 times graphically in the first one second intricately, failing to do the same periodically (Table. 3). Yet this frequent intersection-pattern makes up for the rarity found in super-tonic making the chord mediocre as far as tonal stability is concerned in a diatonic major scale.

\section{Results}

The study analyzes the working of musical chords at the level of frequencies of constituent notes drawing evidences substantiating significant prospects like the resolutive nature of leading-tone, the hypothesis of tonal inertia that go in agreement with the proven principle of active and stable notes. These significant results open up a wider scope for framing chord progressions and to better understand the working of chords and their tonal movements in any given piece scientifically.

\section{References}

1. CNRS (Délégation Paris Michel-Ange). "How does the human brain memorize a sound?." ScienceDaily.

2. Josh McDermott et al, Understanding how the brain makes sense of sound - Massachusetts Institute of Technology - National Science Foundation (2019)

3. Richard Bass, Enharmonic Position Finding and the Resolution of Seventh Chords in Chromatic Music, Music Theory Spectrum, Volume 29, Issue 1, Spring 2007, Pages 73-100, https://doi.org/10.1525/mts.2007.29.1.73

4. Barry Leshowitz and Edward Cudahy, Frequency discrimination in the presence of another tone, The Journal of the Acoustical Society of America 54, 882 (1973); https://doi.org/10.1121/1.1914342

5. W. M. Hartmann, The effect of a leading-tone on the pitch of a sine tone-intensity step experiment, The Journal of the Acoustical Society of America 68, S109 (1980); https://doi.org/10.1121/1.2004537

6. Andrew Motte's English translation: Newton, Isaac (1846), Newton's Principia : the mathematical principles of natural philosophy, New York: Daniel Adee, p. 72

7. David Wright, 2009, Mathematics and Music. ISBN Number: 9780821848739

8. Above All - Lenny LeBlanc; Copyright and/or license held by SME (Sony Music Entertainment).

9. Catherine Schmidt-Jones, 2015, Understanding Basic Music Theory, ISBN Number: 9781508534297

10. Theo. Hoelty-Nickel et al. 1970, The Musical Heritage of the Church Volume VII 
Tables and Figures

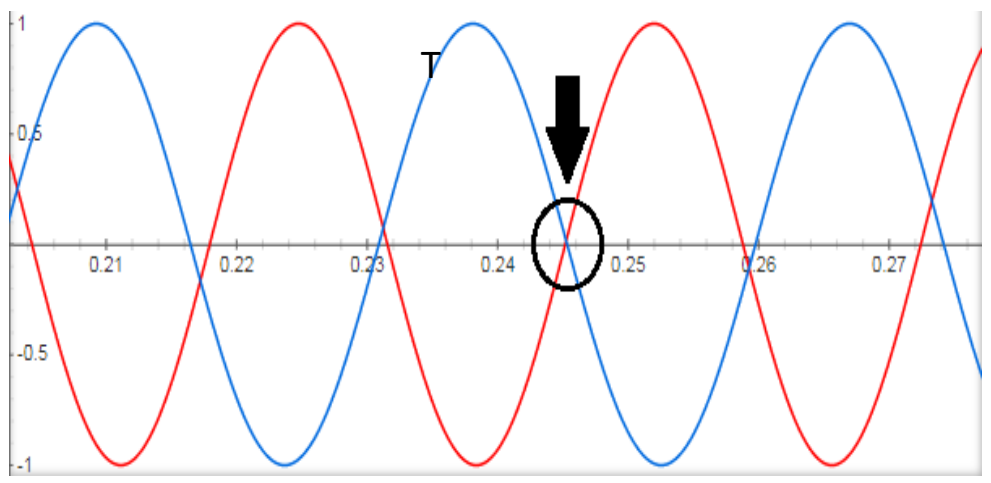

Figure. 1 Intersections in respective graphs of Leading-tone and Tonic (T)
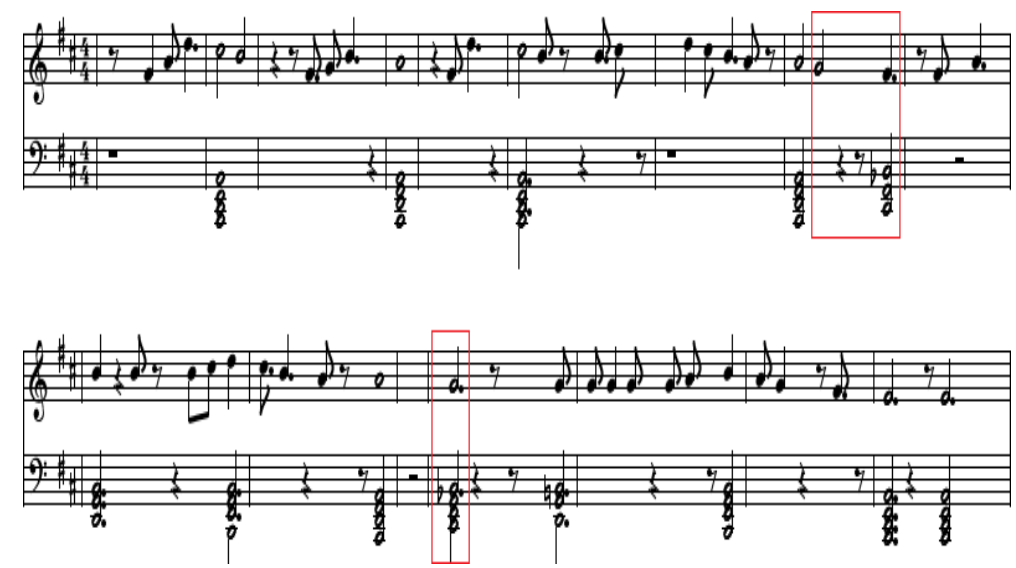

Figure. 2 Verse of the song 'Above All'

\begin{tabular}{|l|l|l|l|l|l|l|l|l|l|}
0.2453 & 0.4906 & 0.7216 & 0.9668 & 1.2121 & 1.457 & 1.702 & 1.948 & 2.179 & 2.4243 \\
\hline
\end{tabular}

Table. 1 Points in time of periodic intersections of graphs of frequencies of Tonic and Leading-tone

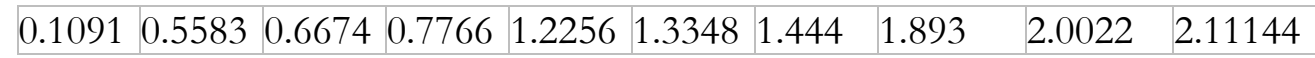

Table. 2 Points in time of intersections of graphs of frequencies of Tonic and Supertonic.

$$
\begin{array}{llllllllll}
0.2591 & 0.29966 & 0.3402 & 0.5588 & 0.59930 .6398 & 0.8584 & 0.8989 & 0.9394 & 1.1582
\end{array}
$$

Table. 3 Points in time of intersections of graphs of frequencies of Tonic and Major 6th. 